\title{
Correlation of water consumption with nutritional status, metabolic risk and percentage of body fat in adults of Mexico City
}

\author{
$\underline{\text { Rey Gutiérrez }}{ }^{1}$, Claudia Radilla ${ }^{1}$, Salvador Vega ${ }^{1}$, Juan Rivera ${ }^{2}$, Jorge Ruiz ${ }^{3}$ and María Radilla ${ }^{4}$ \\ ${ }^{1}$ Autonomous Metropolitan University Campus Xochimilco, Mexico City, Mexico, \\ ${ }^{2}$ Autonomous Metropolitan University Campus Iztapalapa, Mexico City, Mexico, \\ ${ }^{3}$ Autonomous University of Chiapas, Chiapas, Mexico and \\ ${ }^{4}$ Foundation Aprende con Reyhan A.C. Foundation Aprende con Reyhan A.C., Mexico City, Mexico
}

\section{Abstract}

The promotion of water consumption has been identified by the government of Mexico as one of the main strategies to reduce the diet energy density in order to prevent the problems of overweight, obesity and other diseases. The aim of the present study was to determine the correlation of the water consumption with nutritional status, metabolic risk and percentage of body fat in adults of Mexico City. A sample of 12733 adults residing in Mexico City was obtained, of which $75.6 \%(n=9623)$ belongs to the female gender and $24.4 \%(n=3110)$ belongs to the male gender. In the study those adults who were between 21 and 60 years old, with an average age of $39.59 \pm 10.97$ years, were included. The population was constituted in several health fairs celebrated in Mexico City from 2015-2018, in which nutritional consultation was provided. By correlating water consumption with nutritional status, metabolic risk and percentage of body fat, it was found that adults who consume a glass of water of $250 \mathrm{~mL}$ per day, have higher incidence of obesity, metabolic risk and percentage of body fat high $(70.0 \%, 83.6 \%$ and 96.2 , respectively), compared to adults who consume seven glasses of $250 \mathrm{~mL}$ of water per day equivalent to $1750 \mathrm{~mL}$ of water per day $(40.4 \%, 63.8 \%$ and 92.9 , respectively), finding a highly significant difference $(\mathrm{p}<0.01)$. In the present study it can be observed that adults who have optimal water intake have a lower incidence of obesity, metabolic risk and high percentage of body fat, data that agree with the literature. However, despite the fact that the multiple benefits of water intake have been documented, its consumption among the population in general is very low, so it is suggested to continue adding efforts to raise awareness among the population regarding the importance and benefits on health by staying well hydrated. Likewise, it is important to consider that the promotion of water consumption is an important component in strategies to promote healthy habits and lifestyles.

\section{Conflict of Interest}

There is no conflict of interest 